indicates that temperatures are cold enough for the chemical components of planets to form. It also increases the density of dust grains and helps them to clump together. A team led by Chunhua Qi of the Harvard-Smithsonian Center for Astrophysics in Cambridge, Massachusetts, and Karin Öberg of the University of Virginia in Charlottesville studied the gas disk surrounding TW Hydrae, a star 54 parsecs from Earth. This revealed signals of diazenylium, an ion that exists mainly in areas where carbon monoxide is frozen. The team found this snow line about 30 Earth-Sun distances from the star. Knowing the location could help astronomers to shape models of planet formation in the Solar System and beyond, the authors say.

Science http://dx.doi.

org/10.1126/science.1239560 (2013)

\section{CELL BIOLOGY \\ Twists in a protein factory}

A protein-synthesis organelle called the endoplasmic reticulum is shaped like a spiralling car park — and can add new levels as needed.

A team led by Mark Terasaki at the University of Connecticut Health Center in Farmington and Tom Rapoport at Harvard Medical School in Boston, Massachusetts, produced extremely detailed electron microscopy images of the organelle by staining very thin sections using a technique that accentuates the membrane sheets from which it is built. This method revealed sheets that form a twisting, continuous membrane with layers connected by helical ramps. The team described this geometry precisely using mathematical modelling and showed that the structure allows for dense, adjustable packing of material in the cell, boosting the surface available for protein production within a small volume.

Cell 154, 285-296 (2013)
CLINICAL GENOMICS

\section{TB resistance revealed}

DNA sequencing can reveal antibiotic resistance in tuberculosis (TB) infections faster than standard tests.

The causative agent of TB, Mycobacterium tuberculosis, grows so slowly in culture that assessing its susceptibility to antibiotics can take one to two months, with each drug requiring its own test. A team led by Sharon Peacock at the University of Cambridge, UK, used whole-genome sequencing to investigate a case of tuberculosis that had shown resistance to many antibiotics. Within days of sampling and culturing the patient's mucus, sequencing revealed co-infection with two strains of tuberculosis, a condition that had not been detected with standard tests. The team examined genes known to influence the effectiveness of 39 antibiotics. The results indicated that the strains were resistant to nine drugs that had already been identified by a reference laboratory, as well as to five others not yet assessed.

N. Engl. J. Med. 369, 290-292 (2013)

\section{CLIMATE MODELLING}

\section{Ice-free Arctic predicted}

High levels of greenhousegas emissions could drive the annual sea-ice minimum in the Arctic, which occurs in September, to a level defined as ice-free by around midcentury.

A team led by Jiping Liu of the State University of New York in Albany assessed recent projections from 30 climate models on the basis of how well they represent current sea-ice levels. The researchers also looked at the projections of those models that best represented the evolution of sea ice from 1979-2011. Both analyses suggest that, compared with 2012 levels,

COMMUNITY CHOICE

The most viewed papers in science

\title{
Psychotherapy helps depression
}

\section{HIGHLY READ \\ on plosmedicine. org in July}

Extracting robust evidence for the value of psychotherapy from clinical trials has been challenging, but comprehensive analysis of trials has found that various types of

psychotherapy provide similar benefits for depression.

Jürgen Barth from the University of Bern, Switzerland, and his colleagues analysed 198 randomized, controlled clinical studies, covering seven psychotherapeutic techniques and involving 15,118 people with depression. The researchers used a technique called network meta-analysis, which allowed them to extract comparative information from individual studies.

Their analysis showed that each of the psychotherapies helped patients to a similar degree - but the effect was less marked in larger and blinded trials than in smaller and non-blinded ones. The clearest benefits were seen in the large and more rigorous studies assessing cognitive behavioural, interpersonal and problem-solving therapies.

PLoS Med. 10, e1001454 (2013)

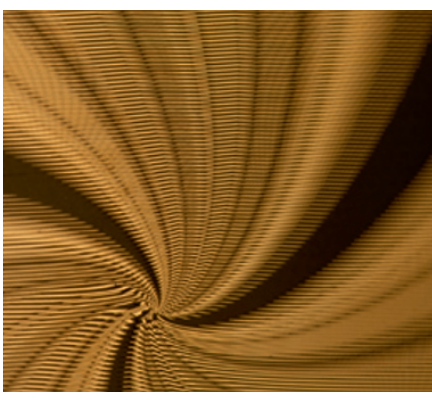

sea ice could decline by some $50 \%$ to around 1.7 million square kilometres by the 2060 s if emissions are moderate. A high-emissions scenario could push the annual minimum to less than 1 million square kilometres - the ice-free level - in the 2050s.

Proc. Natl Acad. Sci. USA http://dx.doi.org/10.1073/ pnas.1219716110 (2013)

\section{MATERIALS SCIENCE}

\section{Illusions to foil counterfeiters}

Microscopic markings that are too small to be reproduced by standard printers can give rise to optical illusions, and could be used to mark authentic banknotes or luxury-brand goods.

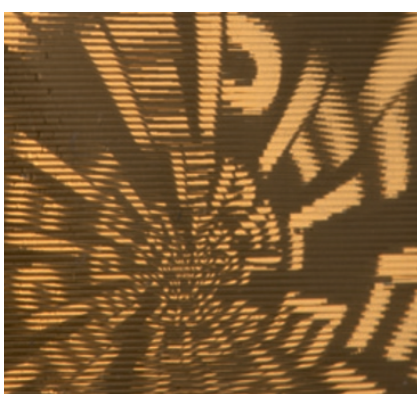

Jürgen Brugger, Victor Cadarso and their group at the Swiss Federal Institute of Technology in Lausanne fabricated microscopic lines in rows or spirals (pictured, left) by patterning pixels in gold using ultraviolet light. When an array of tiny cylindrical lenses is laid over the lines, interference patterns produce images (pictured, right) of letters and other symbols. Copying these patterns, or moirés, would be difficult, the researchers say, because even micrometresized blemishes visibly distort the illusion.

Light Sci. Appl. 2, e86 (2013)

\section{$\rightarrow$ NATURE.COM}

For the latest research published by Naturevisit:

www.nature.com/latestresearch 